\title{
Compact Vibration Measuring System for in-vehicle Applications
}

\author{
G. J. Stein ${ }^{1}$, R. Chmúrny ${ }^{1}$, V. Rosík ${ }^{2}$ \\ ${ }^{1}$ Institute of Materials and Machine Mechanics, Slovak Academy of Sciences, Račianska 75, SK - 83102 Bratislava, \\ Slovakia, e-mail: stein@savba.sk, ummschmu@savba.sk \\ ${ }^{2}$ Institute of Measurement, Slovak Academy of Sciences, Dúbravská cesta 9, SK - 84104 Bratislava, Slovakia, \\ e-mail: umerrosi@savba.sk
}

Low frequency vibrations occurring in ground transportation are of interest in research of the driver's and the passenger's comfort and in seating dynamics research. Piezoelectric accelerometers commonly used for vibration measurement are not well suited for the analysed frequency range. Hence, other sensors capable of measuring accelerations down to the sub-Hertz region have to be used. MEMS acceleration sensors are particularly suitable for such purposes. A compact measuring system employing two three-axial MEMS accelerometers interfaced via a USB data acquisition unit to a laptop was designed and constructed. The digitised data were then processed by scripts in Matlab ${ }^{\circledR}$ with the aim of analysing both the effect of vibration on a seated person and the dynamic properties of the seat. Preliminary results of a test run with a passenger automobile are presented.

Keywords: vibration measurement, MEMS accelerometer, human comfort, driver's seat properties

\section{INTRODUCTION}

$\mathrm{O}$ NE OF THE AREAS in which low frequency vibrations occur is ground transportation. The frequency content may vary from well below $1 \mathrm{~Hz}$ up to $80 \mathrm{~Hz}$. Exposure to low frequency vibrations and shocks may affect human comfort and in some cases prolonged exposition to excessive vibrations may impair health. In many industrial vehicles, forestry and agricultural machinery, building and mining vehicles and machinery, due to their principle of operation, it is not possible to use axis suspension for vibration suppression, as it is the case in passenger cars, buses, railway vehicles, etc. It is necessary to undertake technical measures to minimize the effects of the vibration influence on workers by designing suitable driver's seats. Various national and international regulations limit the vibration exposure of workers. In Europe, this important issue is regulated by the Physical Agents (Vibrations) Directive 2002/44/EC [1]. According to this Directive, there are vibration exposure limits for workers subjected to the whole body vibration for a standardised 8-hour shift, which must not be surpassed. The particulars of measurement of exposition to vibrations are governed by the standard [2].

One of the contemporary approaches to the seat development is the seat suspension system modelling and simulation. Simulation models of various complexities are employed for this task. The models can be excited by artificial signals, e.g., sweep harmonic signal, narrow band random signal or by acceleration time history data gathered in real field operating conditions. The last type of signals serves best for the description of the real operating conditions. Hence, it is important to have suitable instrumentation at hand for the acquisition of the vibration data in field conditions.

Various means for the acquisition of the vibration data in the field conditions are used, i.e. in-vehicle analogue data loggers, telemetry systems, instrumentation grade tape recorders, instrumentation carried in an escort vehicle [3]. Recently, instead of instrumentation grade tape recorders, solid-state memory cards based field recording instruments were introduced by renowned companies [4], [5].

All these approaches are rather complex and costly and are usually limited to the use in specialised test establishments, on test tracks of large vehicle or seat manufacturers, where their extensive daily use is warranted and the capital cost incurred is well justified. Their use in academic research environment is sometimes limited due to the costs, complexity and the need of a knowledgeable specialist fully occupied with the tests. Even so, errors in the field measurements due to miss-connections of the sensors, contact/earthing problems, and electromagnetic interference may spoil the costly and extensive field test results, performed often under heavy and stressful conditions in a short time span available for tests. Quite often, later it is established that some data files are unsuitable for further analysis, say, due to electro-magnetic interference, excessive cross-talk failures of the sensors or of the cabling.

In most cases the vibration transmission from the vehicle floor (seat base) to the seated driver via the driver's seat and the driver's buttocks is in the centre of attention. In general, translatory and rotational vibration components are present; however, the translatory components in mutually perpendicular coordinate axis are the most often measured and evaluated.

Wireless vibration monitors [6], [7] were recently introduced to monitor the real vibration loading of the workers in the field conditions. For attended measurements, companies dealing with the measurement and the analysis of the vibration provide the so-called human vibrometers. These special-purpose vibration measuring instruments have to comply with the ISO 8041:2005 standard [8].

To facilitate the three-axial vibration measurement in field conditions, a low-cost apparatus from the commercially available MEMS acceleration sensors was developed in 
collaboration with the Institute of Measurement of the Slovak Academy of Sciences. The aim behind the project was to have the in-house facilities to perform the field measurements within reasonable costs without the need for the specialised equipment and for the specialised test engineer. The purpose of this system is:

1. To gather real-time field vibration data for the future use in the seat suspension system simulation research.

2. To have the means to analyse the seating dynamics concurrently in three mutually perpendicular axes. In this way, the various interactions could be analysed in each vehicle. The research effort could then focus on the vibration mitigation in the axis of the highest influence (acceleration value). This is not necessarily in the vertical axis.

A contemporary state-of-the-art field vibration acquisition system [9] consists of:

- Tri-axial ICP piezoelectric accelerometer - one mounted under the seat, the other one in a specifically designed seat-pad according to ISO 10326-1:1992 [10], i.e. EN 30326-1:1994 [11] located between the buttocks of the seated test subjects. As described in these standards, it serves to measure the vibratory input with respect to the seated person.

- Two professional human vibrometers (vibration data loggers) for amplifying the accelerometer signals.

- A pocket Personal Digital Assistant (PDA) with an auxiliary battery pack and an extended memory with an external PCMCIA data acquisition card.

- A Global Positioning System (GPS) integrated within the PDA to supply the positional data for use in the laboratory.

The continuous data streams acquired in various test runs in a metropolitan bus were stored in the PDA and downloaded to a PC equipped with LabVIEW after each testing to process the raw collected data according to the standards used in the assessment of the human body vibration influence and in the seating dynamics research, to be described below.

The extent and complexity of the instrumentation used is obvious from this short description. It has to be stressed that, except for the GPS auxiliary data, only four channels of vibration data were processed by the professional human vibrometers working as accelerometer conditioning amplifiers for the PCMCIA data acquisition card. It was possible to analyse the vertical vibration transmission only. Vibration transmission in other directions was not analysed. Given the large number of performed test runs, statistical analysis of the raw data was possible, as described in detail in [9]. The aim of this extensive study was to thoroughly characterise and select the best of the three different driver's seats tested.

Even more sophisticated instrumentation apparatuses were used in the field test made within the European project with the acronym VIBSEAT [12], described in a greater detail in the relevant field test reports. Some of the large-scale measurements in difficult conditions were not very conclusive either. Hence, it is necessary and worthwhile to pay attention to the improvement of methods and devices for gathering of field vibratory data for laboratory analysis and evaluation, as well as an excitation signal for simulation.

\section{SPECIFICS OF THE VIBRATION MEASUREMENT IN TRANSPORT MEANS}

It should be noted that in the ground transportation the acting forces are of primary interest, followed by the relative movement of various vehicle parts. As a result, the preferred measurand is the absolute acceleration followed by the relative displacement. Despite a well-defined physical relation between both characteristics, experience shows [13]-[15] that the errors introduced by the real measuring systems are of such a magnitude that excludes the practical usage of this relation. In practical situations, it is advisable to complement one measuring system by another one and to use data fusion [13].

It is known from experience that the vibratory acceleration $a_{\mathrm{v}}$ observed in the ground transportation is smaller than standard gravity acceleration $\mathrm{g}_{\mathrm{N}} \approx 9.81 \mathrm{~m} \mathrm{~s}^{-2}$. It is also well known that the measured accelerations are of random nature with periodic components. Often, shocks are encountered, the peak acceleration magnitude which may be as large as $30-40 \mathrm{~m} \mathrm{~s}^{-2}$ (3- to 4-times $\mathrm{g}_{\mathrm{N}}$ ), gives rise to crest factors of the excess of eight. Vehicles are, in general, subjected to traverse on arbitrary curvilinear trajectory with non-constant traversing velocity. Moreover, the vehicle chassis plane may be inclined with respect to the horizontal plane. Both effects give rise to a quasi-static acceleration $a_{\mathrm{t}}$, which is superimposed onto the mechanical vibrations due to the road/track unevenness and/or the engine influence. The quasi-static acceleration influences the equilibrium position of the seat suspension system and so it is of interest in the seating dynamics research: A seat is a non-linear oscillatory system, whose properties strongly depend on the operating point in the equilibrium. Thus, it is worthwhile to posses a measuring system that is able to measure both acceleration components.

The quasi-static translatory acceleration $a_{\mathrm{t}}$ frequency content extends down to the sub-Hertz frequencies. Measurement of the total acceleration $a_{\mathrm{T}}=a_{\mathrm{t}}+a_{\mathrm{v}}$ poses some practical problems, as treated at length, e.g., in [13], [14]. The use of the standard piezoelectric accelerometers with their well-known inherent low frequency limits is not feasible. Other accelerometer types, capable of measuring the quasi-static acceleration have to be considered. The current state of the art is to use sensors based on the socalled servo-accelerometer principle and produced using contemporary semiconductor manufacturing technologies, i.e. Micro-Electro-Mechanical Systems (MEMS), as described, e.g., in [13], [16]-[18]. The use of one type of the MEMS type servo-accelerometer for this purpose will be illustrated further on. A detailed description of this sort of acceleration sensor may be found in the above-mentioned references. Some information on the noise issues pertinent to the MEMS accelerometers is given in [19], [20]. 


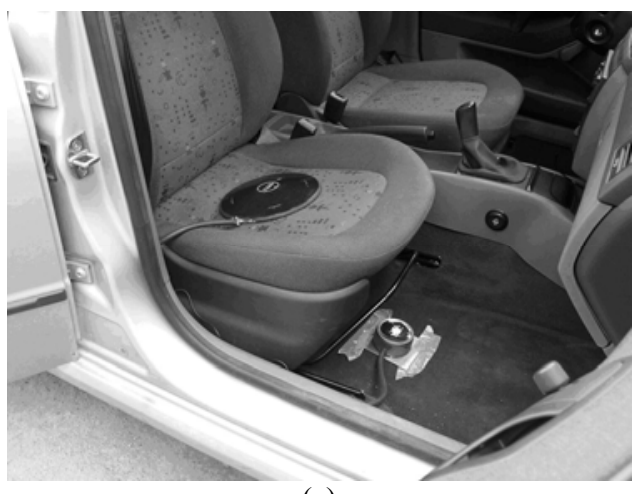

(a)

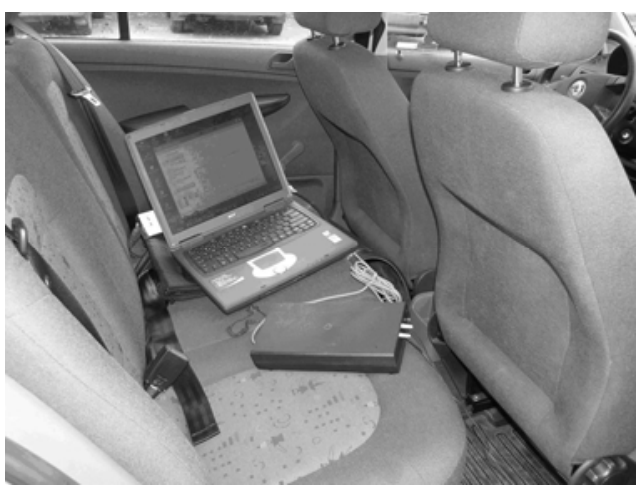

(b)

Fig.1. Photo of the sensors (a) and the box with the laptop (b).

\section{THE COMPACT MEASUREMENT SYSTEM}

The compact vibration measuring system consists of the following parts (see Fig.1):

A/ Two identical three-axial MEMS accelerometers;

B/ An analogue acquisition unit with a USB output;

$\mathrm{C} / \mathrm{A}$ standard laptop with an acquisition program.

A/ The three-component MEMS accelerometer type CXL04LP3, made by Crossbow [21], is enclosed in a plastic box $19 \times 47.6 \times 25.4 \mathrm{~mm}$ of the mass of $46 \mathrm{~g}$. The measuring range is $\pm 40 \mathrm{~m} \mathrm{~s}^{-2}\left(4 \times g_{\mathrm{N}}\right)$; the output voltage is approx. $2.5 \mathrm{~V}$ for the zero acceleration and sensitivity is $\approx 50 \mathrm{mV} / \mathrm{m} \mathrm{s}^{-2}$. According to the manufacturer, the frequency range extends from $\mathrm{DC}$ to $f_{\mathrm{M}}=100 \mathrm{~Hz}$. The accelerometer is factory calibrated; hence no field calibration is needed.

The accelerometer is fixed either to a $2 \mathrm{~mm}$ thick steel plate of $120 \times 100 \mathrm{~mm}$ (the base/floor sensor, denoted as "sensor A" (index "b")) or to a steel disk of a diameter of 70 $\mathrm{mm}$, located in a rubber disc "sensor B" (index "s"); Fig.1(a). The rubber disc is made according to the requirements of ISO 10326-1 standard, i.e. EN 30326-1 [10], [11] (see Fig.2). No compensation of the temperature variation was foreseen because a high-pass filter eliminates any incurred low frequency drift.

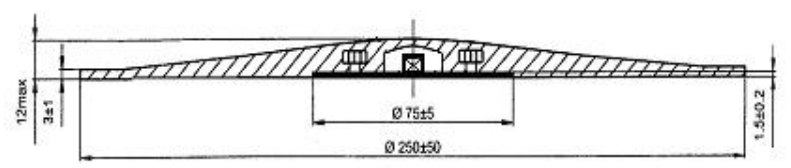

Fig.2. The test disc to be positioned under the seated driver.

$\mathrm{B} /$ The analogue acquisition unit with USB 2.0 communication interface, type DT 9816, made by Data Translation [22] consists of 8 input channels, each equipped with 16-bit sigma-delta convertors with sampling frequency of up to $50 \mathrm{kHz}$ and a full span of $\pm 5 \mathrm{~V}$. The 8 channels are timed simultaneously (aperture match better than $5 \mathrm{~ns}$ ), thus facilitating reliable phase/time delay estimation. The control is facilitated by a microcontroller. The acquisition software enables sampling of either 32000 data points into a memory file or a continuous storage of a much larger amount of data on the disk. For chosen sampling frequency $f_{\mathrm{s}}$ of $200 \mathrm{~Hz}$, the first method facilitates measurement duration of $150 \mathrm{~s}$, which was deemed as sufficient at this stage.

Compact integration of the above-mentioned units was facilitated. Since the sensors rely on a constant voltage supply of $5.0 \mathrm{~V}$ with required stability, a stabilisation circuit of ADP 3607 type was used. To increase the system reliability no connectors were used. The cables of the sensors and the USB cable enter the compact box via rubber bushings, thus facilitating a partial dust-proofness, Fig.1(b). These measures improve the reliability of the device and simplify the measurement set-up in the field.

To make the experimental conditions more transparent, a straight traverse is assumed. This condition calls for a specific field measurement conduct: the vehicle stands in a standstill with the engine running for a while, while the data acquisition is already running, then accelerates to the velocity required, maintains a straight course at a given constant velocity and then decelerates to the standstill. This measurement organisation facilitates proper data analysis for the seat suspension system performance assessment in the equilibrium position.

C/ The data analysis program is written in the Matlab ${ }^{\circledR}$ environment. The program first acquires the raw data, including the interval at the beginning of the measurement when the vehicle is at a standstill. The signal is subjected to a low-pass filtration by a FIR low-pass filter of the $100^{\text {th }}$ order with a cut-off frequency of $0.2 \mathrm{~Hz}$. In this way, the translatory acceleration $a_{\mathrm{t}}$ is obtained and displayed (Figs.3(a), 3(b)). The program calculates the sensor inclination angles, which may be of use in seat design. In the illustrative example, the most pronounced inclination is for the seat sensor B in the fore-and-aft direction (some $14^{\circ}$, causing the observed offset), whereas the other values are about $2^{\circ}$ and hence negligible. These DC components are then subtracted from the time sequence to provide a correction for sensor inclination and temperature drift.

The operator can assess from Fig. 3 the time interval when the vehicle was travelling with a constant speed (base $a_{\mathrm{t}}$ in the fore-and-aft direction is approx. zero). In the next step, the time interval during which the signal is assumed to be stationary is selected. In this example, this is between the $32^{\text {nd }} \mathrm{s}$ and the $38^{\text {th }} \mathrm{s}$. 


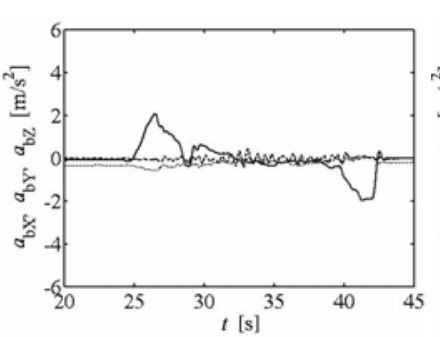

(a)

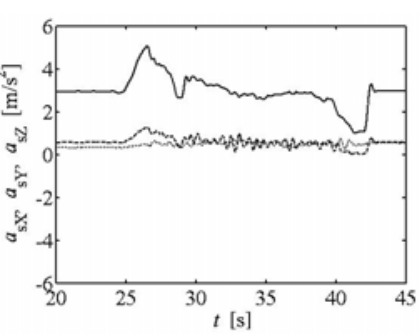

(b)
Fig.3. Pre-processing of the acceleration signals: (a) on the seat base; (b) and on the seat surface: $(-) x$ - direction; $(\cdots .)$.$y - direction; (---) upward direction ( z$ - direction).
Signals from the selected time interval are then subjected to a further band-pass filtration by a FIR filter of the $100^{\text {th }}$ order to extract the vibratory acceleration $a_{\mathrm{v}}$ in the frequency band $0.50 \mathrm{~Hz}$ to $80 \mathrm{~Hz}$ in each sensor axis (Fig.4), in accord with the ISO 8041 standard [8]. The data preprocessed like this are subjected to calculation of the acceleration power spectral densities $\left(\mathrm{PSD}_{\mathrm{ij}}\right)$, modules of the transfer function estimates $\left(\mathrm{TFE}_{\mathrm{j}}\right)$ and respective coherence functions $\gamma_{\mathrm{j}}$ by Matlab ${ }^{\circledR}$ functions, Fig.5. This is the standard time history data processing used in the analysis of mechanical vibrations.

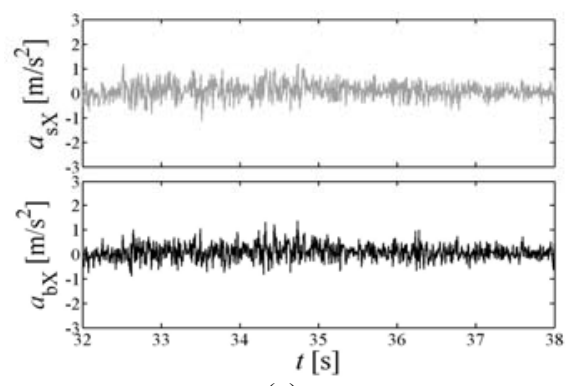

(a)

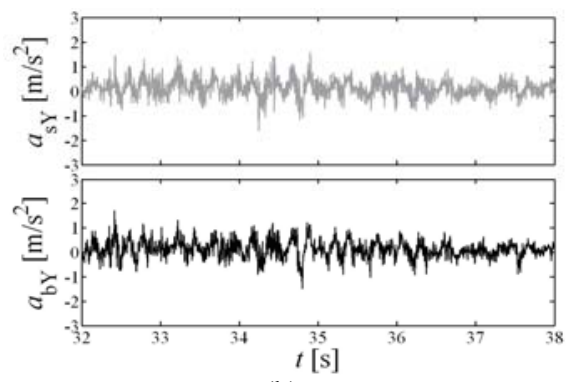

(b)

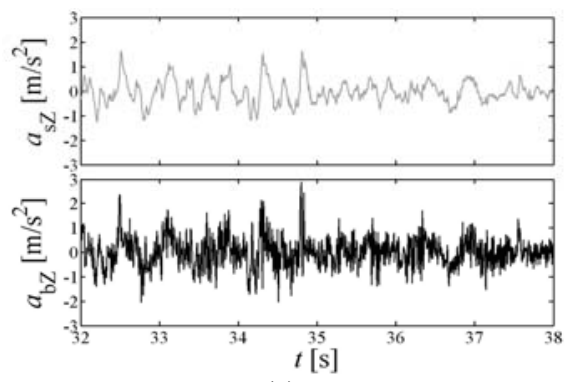

(c)

Fig.4. Vibratory acceleration signals in mutually perpendicular axes: (a) $x$, (b) $y$, (c) $z$; seat base (—), seat (—).

\section{PRELIMINARY RESUlTS}

The data used for the performance assessment were gathered in preliminary test runs with three voluntary test persons seated in a Škoda sedan car on the premises of the Slovak Academy of Sciences. Due to the limitations of the straight traverse with a constant speed in the test area, analysed time intervals are very short and the traversing speed rather low (some 10 to $15 \mathrm{~km} / \mathrm{h}$ ). No test track closed to public traffic was available; hence more field tests were not yet possible.

The acceleration RMS values were low; however, still more than $12 \mathrm{~dB}$ above the sensor's noise floor. For estimation of the acceleration power spectral densities $\left(\mathrm{PSD}_{\mathrm{ij}}\right)$, transfer function estimates $\left(\mathrm{TFE}_{\mathrm{j}}\right)$ following parameters for the FFT routine were used: $M=512$ data points, i.e. frequency resolution $\Delta f=0.391 \mathrm{~Hz}, 50 \%$ overlap of adjacent data blocks and Hanning weighting, Fig.5. Due to short time history, the number of averaged blocks ranged between 2 and 5 .

\section{DISCUSSION}

The described compact vibration measuring system for the in-vehicle applications should fulfil different purposes. It was developed for the specific use in the academic research environment with limited means, not for industrial use on test tracks. The vibration measuring system was not calibrated and tested to comply with the ISO 8041 standard, as this was not required. The authors fully relied on the sensor calibration data supplied by the sensor manufacturer.

On the other hand, the compact measuring system fully utilises the available hardware, unlike the instrumentation used, e.g., by [9]. The software can be tailored to the specific tasks, i.e. to the driver's seat performance analysis or for the evaluation of vibration influence on the seated driver or for the field vibratory data acquisition.

There is a need of a person to operate the laptop during data acquisition. However, the data acquisition is quite simple and does not require sophisticated skills. Thus, a trained driver is able to perform the recordings by himself. When using a human vibrometer supplied by a specialist company, the measurement technician has to travel on the machine tested and operate the vibrometer by himself, anyhow. This may psychologically adversely influence the driver's performance and hence the measured results. However, the circumstances of the particular measurements can be minutely recorded to be used later in laboratory, e.g., for comparison of simulation results with those measured. This is often of a paramount importance. Moreover, the presence of the test technician enables to identify erroneous measurements and repeat the respective test run without much extra effort on the spot, directly in the field.

Presence of another person is nearly completely eliminated by wireless systems [6], [7] which are designed for a longterm unattended operation. However, they gather just the RMS acceleration values, i.e. time integrals of the accelerations in the three mutually perpendicular directions, not the raw acceleration signals. Hence, the stored data are not suitable for supplying any simulation program. The same is true for the professional human vibrometers. None of these instruments are suitable for acquisition and storage of the data required for detailed scientific research.

This is possible with the described compact vibration measuring system and with the apparatus used in the references [3], [9]; but at a higher cost. 

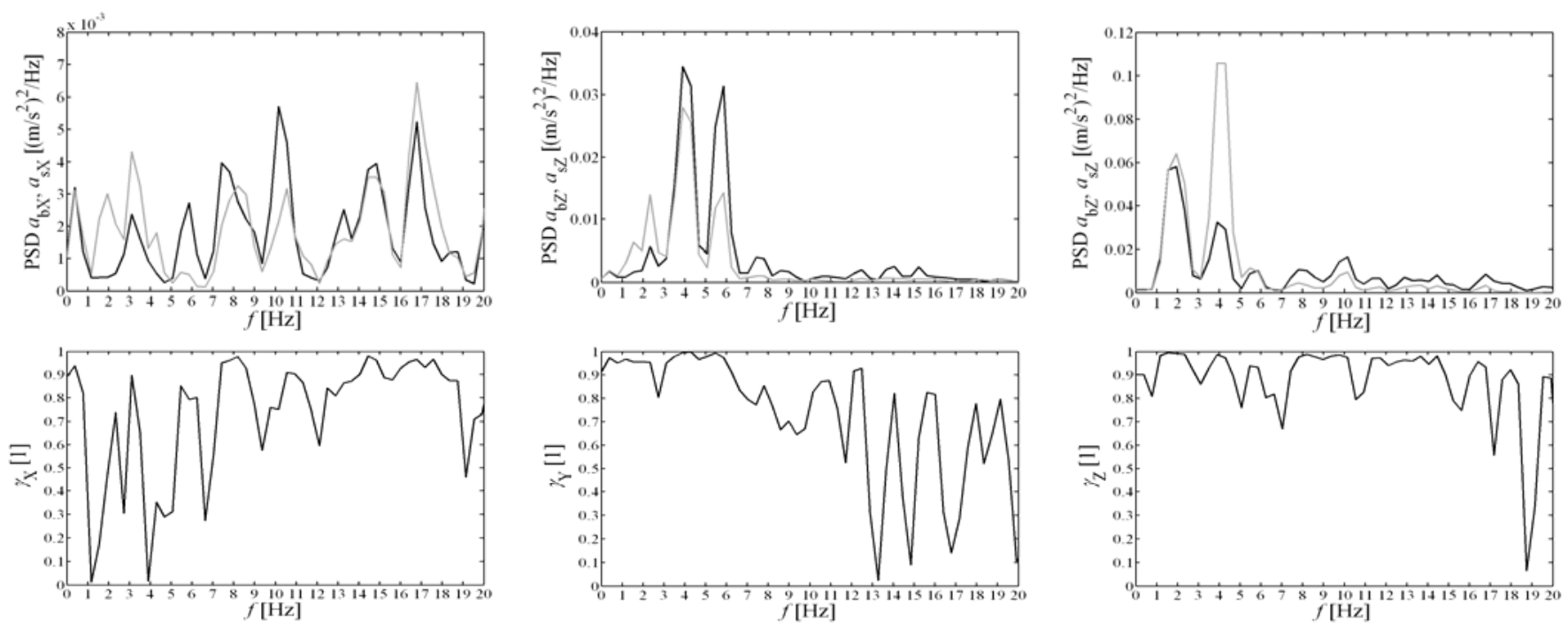

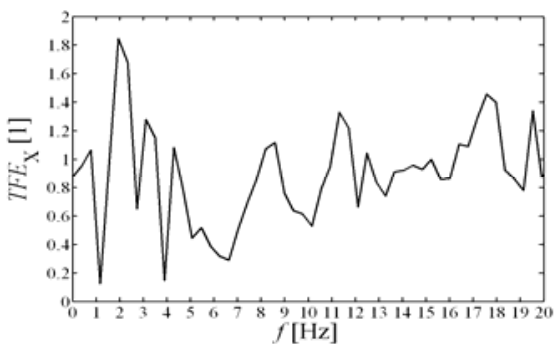

(a)

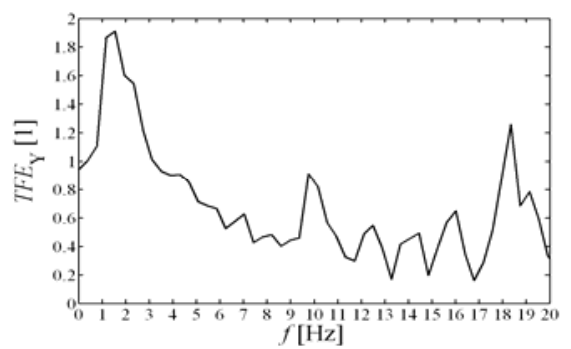

(b)

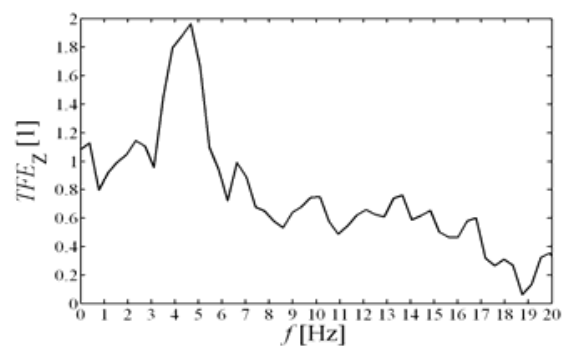

(c)

Fig.5. Typical seat base and seat surface acceleration PSDa, the coherence functions $\gamma$ and the TFEs for the three perpendicular axes: (a) $x$, (b) $y$, (c) $z$; seat base (—), seat (-).

\section{CONCLUSIONS}

To facilitate the three-axial vibration measurement in the field conditions, an apparatus from commercially available MEMS acceleration sensors was developed. The aim was to have instrumentation for field measurements of vibrations at a reasonable cost without the need of specialised equipment. The use of this system is twofold:

1. To gather the field vibration data in real time for future use in a seat suspension system simulation research.

2. To have the means to analyse driver's seat performance in the three mutually perpendicular axes.

The developed system fully utilises the hardware used and seems to be well suited for the academic type of research. The fitting of the apparatus into the vehicle is fairly easy and straight forward, as no cable connections are necessary. By suitable programming in the laboratory, the gathered field data can be processed according to the tasks required. Preliminary results demonstrate the feasibility and the capabilities of the described measuring system.

\section{ACKNOWLEDGMENT}

The article was prepared within projects No. 2/6161/26 and No. 2/0075/10 of the Slovak Grant Agency for Science VEGA. The support is gratefully acknowledged.

\section{REFERENCES}

[1] European Commission. (2002). Directive 2002/44/EC of the European Parliament and of the Council of 25 June 2002 on the minimum health and safety requirements regarding the exposure of workers to the risks arising from physical agents (vibration). Official Journal of the European Communities, L 177, 13-19.

[2] European Committee for Standardisation. (2007). Mechanical Vibration - Measurement and Calculation of Occupational Exposure to Whole-Body Vibration with Reference to Health-Practical Guidance. EN 14253:2003+A1:2007. Brussels, Belgium.

[3] Pinto, I., Stacchini, N. (2006). Uncertainty in the evaluation of occupational exposure to whole-body vibration. Journal of Sound and Vibration, 298 (3), 556-562.

[4] Bruel \& Kjaer Sound \& Vibration Measurement A/S. (2009). Stand-Alone Recorder - LAN-XI-Notar. http://www.bksv.com

[5] RION Co. Ltd. (2009). 4/8-Channel Data Recorder DA-20/40. www.rion.co.jp/english

[6] Wölfel Meßsyteme Software GmbH + Co. KG (2009). EVEC Prospectus. www.woelfel.de 
[7] Marjanen, Y. (2006). Long-term measurements and analysis of day-to-day variability on whole body vibration exposure levels in work environments. In Proceedings of the $13^{\text {th }}$ Intl. Congress on Acoustics and Vibration, 2-6 July, 2003. Vienna, Austria, 887-891.

[8] International Organization for Standardization. (2005). Human Response to Vibration - Measuring Instrumentation. ISO 8041. Geneva, Switzerland.

[9] Blood, R.P., Ploger, J.D., Yost, M.G., Ching, R.P., Johnson, P.W. (2010). Whole body vibration exposures in metropolitan bus drivers: A comparison of three seats. Journal of Sound and Vibration, 329 (1), 109-112.

[10] International Standards Organisation. (1992). Mechanical Vibration - Laboratory Method for Evaluating Vehicle Seat Vibration - Part 1. ISO 10326-1. Geneve, Switzerland.

[11] European Committee for Standardisation. (1994). Mechanical Vibration - Laboratory Method for Evaluation Vehicle Seat Vibration - Part 1. EN 30326-1. Brussels, Belgium.

[12] Human Factors Research Unit, Institute of Sound and Vibration, University of Southampton. (2005). EU Projects VIBSEAT. www.humanvibration.com/ humanvibration/EU/vibseat.html

[13] Doebelin, E.O. (2003). Measurement Systems Application and Design (5th ed.). New York, USA: McGraw Hill.
[14] Webster, J.G. (Ed.) (2001). Acceleration measurement. In Wiley Encyclopedia of Electrical and Electronic Engineering. New York: John Wiley \& Sons, vol. 1, 7-13.

[15] Stein, G.J., Chmúrny, R., Rosík, V. (2007). Measurement and analysis of low frequency vibration. Measurement Science Review, 7 (4), 47-50.

[16] Bernstein, J., Miller, R., Kelley, W., Ward, P. (1999). Low-noise MEMS vibration sensor for geophysical applications. Journal of MEMS, 8 (4), 433-438.

[17] Doescher, J. (1999). A High Performance Surface Micromachined Accelerometer. Technical Note. Norwood, Massachusetts, USA: Analog Devices Inc.

[18] Bernstein, J. (2003). An overview of MEMS inertial sensing technology. Sensors Magazine, February 1, 2003.

[19] Gabrielson, T.B. (1995). Fundamental noise limits for miniature acoustic and vibration sensors. Journal of Vibration and Acoustics, 117 (4), 405-410.

[20] Harley, J.A., Kenny, T.W. (2000). 1/F noise considerations for the design and process optimisation of piezoresistive cantilevers. Journal of MEMS, 9 (2), 226-235.

[21] Crossbow Technology Inc. (2005). Crossbow CLX Series Datasheet. www.xbow.com/index.html

[22] Data Translation Inc. (2005). DT 9816 Prospectus. http://www.datatranslation.com

Received July 27, 2011. Accepted October 21, 2011. 